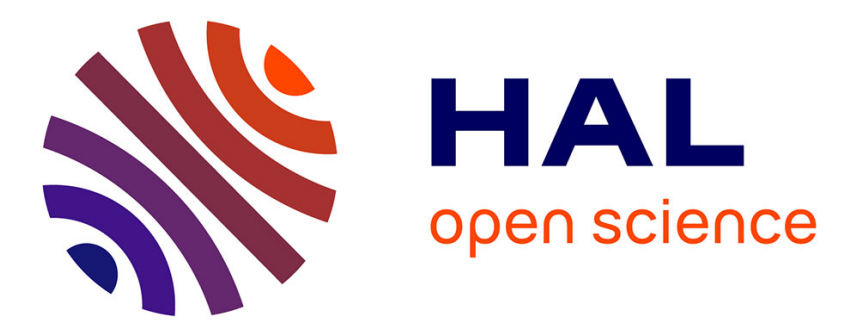

\title{
Theoretical analysis of the DAMAS algorithm and efficient implementation of the Covariance Matrix Fitting method for large-scale problems
}

\author{
Gilles Chardon, José Picheral, François Ollivier
}

\section{- To cite this version:}

Gilles Chardon, José Picheral, François Ollivier. Theoretical analysis of the DAMAS algorithm and efficient implementation of the Covariance Matrix Fitting method for large-scale problems. Journal of Sound and Vibration, 2021, 508, pp.116208. 10.1016/j.jsv.2021.116208 . hal-03225678

\section{HAL Id: hal-03225678 \\ https://hal.science/hal-03225678}

Submitted on 2 Dec 2021

HAL is a multi-disciplinary open access archive for the deposit and dissemination of scientific research documents, whether they are published or not. The documents may come from teaching and research institutions in France or abroad, or from public or private research centers.
L'archive ouverte pluridisciplinaire HAL, est destinée au dépôt et à la diffusion de documents scientifiques de niveau recherche, publiés ou non, émanant des établissements d'enseignement et de recherche français ou étrangers, des laboratoires publics ou privés. 


\title{
Theoretical analysis of the DAMAS algorithm and efficient implementation of the Covariance Matrix Fitting method for large-scale problems
}

\author{
Gilles Chardon* \\ Université Paris-Saclay, CNRS, CentraleSupélec, Laboratoire des signaux et systèmes, 91190, Gif-sur-Yvette, France \\ José Picheral \\ Université Paris-Saclay, CNRS, CentraleSupélec, Laboratoire des signaux et systèmes, 91190, Gif-sur-Yvette, France
}

François Ollivier

Sorbonne Université, CNRS, Institut Jean Le Rond d'Alembert, UMR 7190, F-75005 Paris, France

\begin{abstract}
Based on a theoretical analysis of the DAMAS algorithm, proposed by Brooks and Humphreys to locate and quantify acoustic sources accurately, the paper proposes an efficient method to converge faster to the same solution by implementing standard proven algorithms. We prove that when the DAMAS converges, its limit is a solution of the Covariance matrix Fitting method, and that when the solution is unique, the DAMAS algorithm converges. We analyze the properties of the solutions to this optimization problem to explain the ability of the DAMAS algorithm to recover sparse distributions of sources, even without a regularization term. A fast implementation of the Covariance Matrix Fitting problem is also proposed. Several algorithms to solve this problem are compared. From this review, it comes that the proposed method reduces drastically memory use and computational time thus allowing to address large scale problems. An application to a large-scale 3D problem using experimental data demonstrates this numerical efficiency, and simulations are used to assess the performances of source power estimation.
\end{abstract}

\section{Introduction}

Since its introduction by Brooks and Humphreys, the DAMAS algorithm [1] has enjoyed a certain popularity (see, e.g. [2-4]) in the acoustical imaging community for the deconvolution of beamforming maps (DAMAS stands for Deconvolution Approach for the Mapping of Acoustical Sources). Indeed, it has been observed that when it converges, the DAMAS algorithm yields accurate and useful power estimates [2, 3, 5]. Its development was based on the idea of the deconvolution of the beamforming map under non-negativity constraints. Although not based on firm theoretical foundations, the DAMAS algorithm yields accurate estimations of the spatial extension and powers of acoustical sources. In particular, the DAMAS algorithm is efficient for the imaging of spatially sparse sources, although it does not involve regularization penalties such as the popular $\ell_{1}$-norm.

Still, the DAMAS algorithm has the disadvantage of slow convergence and large memory usage $[3,5]$. Indeed, the algorithm requires the computation and storage of a square matrix of size the number of points in the discretization grid of the space, which can grow quickly, in particular for 3D grids.

\footnotetext{
${ }^{*}$ Corresponding author

Email addresses: gilles. chardon@centralesupelec.fr (Gilles Chardon), jose.picheral@centralesupelec.fr (José Picheral), francois.ollivier@sorbonne-universite.fr (François Ollivier)
}

Several methods, most of them numerically more efficient than the DAMAS algorithm, have been proposed since 2006 (see [2] for a review of such methods), and are frequently compared with it, one recurring remark being the computational effort that the DAMAS algorithm requires [3-12]. Among these methods, several variants of the DAMAS algorithm with improved numerical efficiency have been proposed, based on the formulation of the problem in the Fourier domain [13], or pruning of the grid points that will not be involved in the final source map either directly $[10,11]$, or similarly in a wavelet basis [8]. In the former case, formulation in the Fourier domain is limited to specific geometries, while in the latter, even after pruning, the computational complexity remains too high for large scale problems.

Despite the convincing results of the DAMAS algorithm and its popularity, to our knowledge, no theoretical analysis explains its performances. This article is concerned with the theoretical analysis of the DAMAS algorithm. As described in [1], the DAMAS algorithm consists of a modified GaussSeidel method, where iterates are thresholded to remain positive. In the case where it converges, the limit of the algorithm is necessarily a fixed point of a thresholded Gauss-Seidel iteration. Using this necessary condition, an alternative characterization of the fixed points is given, which can be interpreted as the Karush-Kuhn-Tucker conditions for a non-negative convex quadratic program. This convex problem is shown to be 
equivalent to a non-negative least-squares problem, and a leastsquares fit of a diagonal covariance matrix to the data. A sufficient condition of uniqueness of the solution of these problems can be easily checked. In that case the DAMAS algorithm is shown to converge towards this solution. Microphone selfnoise can be taken into account by removing the diagonal of the spatial covariance matrix, or by explicitly estimating the noise level at each element of the array.

Based on this new characterization of the fixed points (i.e., possible limit points) of the DAMAS algorithm, the use of several optimization algorithms is considered, such as the interior point algorithm [14], the Lawson-Hanson (LH) algorithm for non-negative least-squares [15], and the coordinate descent algorithm [16], of which DAMAS is a particular case. Application to experimental data shows that faster convergence and lower memory usage can be achieved using the Lawson-Hanson algorithm, combined with a factorization of the matrix involved in the objective function. As shown here with experimental measurements, due to the reduced memory usage and computational time, the proposed method outperforms the DAMAS algorithm and allows imaging with larger grids, such as a 3D region. The performances of the proposed method are also analyzed for the estimation of the sources powers using MonteCarlo numerical experiments.

This article is intended to be a self contained analysis of the DAMAS algorithm. While comparison of DAMAS performances with other methods is not its goal, similarities and differences with methods from the literature will be discussed when relevant. The code and data used to generate the numerical results and figures are available online [17].

The article is organized as follows: the DAMAS problem and algorithm are recalled in section 2 . In section 3 , the characterization of the fixed points of the DAMAS algorithm as solutions to convex optimization problems are derived, and convergence of DAMAS is proven under condition of unicity of their solution. Optimization algorithms and implementation details are considered in section 4. Application to experimental data is demonstrated in section 5, and numerical results on the performances of the method are given in section 6 . Concluding remarks are given in section 7 .

\section{Model and DAMAS algorithm}

This section recalls the DAMAS problem and the algorithm to solve it as it was originally proposed in [1]. Acoustic signals are collected on an array of $M$ microphones, and sources are assumed to be located on a discrete grid of size $N$. Usually, the distribution of sources is assumed to be sparse, that is, most of the possible sources are inactive. Measurements are processed in the frequency domain.

\subsection{Model}

Complex amplitudes of the measurements are taken at several times $t_{\ell}$. With $s_{j \ell}$ the complex-valued amplitude of the $j$-th source at time $t_{\ell}$, the measured signals are contained in the $M$ elements vector $\mathbf{x}_{\ell}$ that can be written as:

$$
\mathbf{x}_{\ell}=\sum_{j=1}^{N} \mathbf{d}_{j} s_{j \ell}+\mathbf{e}_{\ell},
$$

where $\mathbf{d}_{j}$ is the Green's function from the $j$-th source to the microphones, $\mathbf{e}_{\ell}$ is an additive noise, assumed white in time and space and uncorrelated with the signals $s_{j \ell}$.

Let $\mathbf{C}$ be the empirical estimate of the data covariance matrix (or spatial covariance matrix, $\mathrm{SCM}$ ) from $L$ snapshots taken at times $t_{\ell}$ :

$$
\mathbf{C}=\frac{1}{L} \sum_{\ell=1}^{L} \mathbf{x}_{\ell} \mathbf{x}_{\ell}^{H} .
$$

Assuming that the signals $\mathbf{s}_{j \ell}$ are independent in time and space, for large $L$ the sample covariance matrix $\mathbf{C}$ converges towards the covariance matrix of the measurements

$$
\mathbf{R}=\sum_{j=1}^{N} p_{j} \mathbf{d}_{j} \mathbf{d}_{j}^{H}+\Sigma
$$

where $p_{j}$ is the power of the $j$-th source and $\Sigma$ is the noise covariance matrix, assumed to be diagonal. With $\mathbf{D}$ the $M \times N$ matrix of columns $\mathbf{d}_{j}$ and $\mathbf{Q}$ the covariance matrix of the signals $\mathbf{s}_{j}$ (assumed to be diagonal with diagonal coefficients $p_{j}$ ), the covariance matrix $\mathbf{R}$ is decomposed as

$$
\mathbf{R}=\mathbf{D Q} \mathbf{D}^{H}+\Sigma .
$$

An estimation of the power value at each grid point can be provided by beamforming: the vector $\mathbf{b}$ is a $N$-elements vector containing the values of the beamforming map, with value at index $i$ given by:

$$
b_{i}=\mathbf{d}_{i}^{H} \mathbf{C} \mathbf{d}_{i} .
$$

Although its formulation is simple, limitations of beamforming are well-known. In particular, closely spaced sources cannot be resolved by beamforming.

Remark 1. When beamforming is directly used for source power estimation, equation (5) is replaced by

$$
\tilde{b}_{i}=\frac{1}{\left\|\mathbf{d}_{i}\right\|_{2}^{4}} \mathbf{d}_{i}^{H} \mathbf{C} \mathbf{d}_{i} .
$$

to obtain meaningful power values. Here, we use unnormalized steering vectors, as the power levels will be obtained after inversion of the beamformed field by solving the DAMAS problem.

When the SNR is high, the noise covariance matrix $\Sigma$ can be neglected. Moreover, for high numbers of samples $L$, the sample covariance matrix $\mathbf{C}$ can be approximated by the covariance matrix $\mathbf{R}$, and the beamforming values are given as a function of the source powers $p_{j}$ by

$$
b_{i} \approx \sum_{j=1}^{N} p_{j}\left|\mathbf{d}_{i}^{H} \mathbf{d}_{j}\right|^{2},
$$


or equivalently as:

$$
\mathbf{b} \approx \mathbf{A p},
$$

where the coefficients of the $N \times N$ matrix $\mathbf{A}$ are given by

$$
A_{i j}=\left|\mathbf{d}_{i}^{H} \mathbf{d}_{j}\right|^{2}
$$

Given $\mathbf{b}$ and $\mathbf{A}$, the DAMAS inverse problem aims at estimating the spatial distribution of sources $\mathbf{p}$ by inverting equation (8).

Remark 2. The matrix A is positive semi-definite. Indeed, it can be written as

$$
\mathbf{A}=\left(\mathbf{D}^{H} \mathbf{D}\right) \odot \overline{\left(\mathbf{D}^{H} \mathbf{D}\right)},
$$

where $\odot$ denotes the Hadamard (component-wise) product and - component-wise complex conjugation. Since $\mathbf{D}^{H} \mathbf{D}$ and its conjugate are both Hermitian semi-positive, $\mathbf{A}$ is positive semidefinite by the Schur product theorem [18]. Moreover, the rank of $\mathbf{A}$ is bounded by the square of the rank of $\mathbf{D}$ [19]. As the dimension of $\mathbf{D}$ is $M \times N$, the rank of $\mathbf{A}$ is bounded by $\min \left(M^{2}, N\right)$. In particular, $\mathbf{A}$ is singular when the size of the discretization grid $N$ is larger than the number of sensors squared $M^{2}$.

\subsection{The DAMAS algorithm}

The DAMAS algorithm takes as input a beamforming map, and returns an estimation of the sources power, assumed to be located on a discrete grid.

In practice, the estimate $\mathbf{C}$ of the covariance matrix is affected by measurement noise, which adds a diagonal matrix, and statistical fluctuations. Moreover, the matrix $\mathbf{A}$ is most of the time ill-conditioned, or even singular (see Remark 2), and naive inversion of (8) does not yield viable results, e.g. negative estimated powers.

Starting from the Gauss-Seidel method for solving linear systems, Brooks and Humphreys proposed the DAMAS algorithm [1], which consists of the Gauss-Seidel algorithm, modified to threshold the iterates to remain positive. The result is, in a sense which was not made explicit, an approximate solution to (8) with non-negative values.

The iterates $\mathbf{p}^{k}$ are obtained by choosing a index $i_{k}$, setting $p_{j}^{k}=p_{j}^{k-1}$ for $j \neq i_{k}$, and

$$
p_{i_{k}}^{k}=\max \left(0, \frac{1}{A_{i_{k} i_{k}}}\left(b_{i_{k}}-\sum_{l=0}^{i_{k}-1} A_{i_{k} l} p_{l}^{k-1}-\sum_{l=i_{k}+1}^{N} A_{i_{k} l} p_{l}^{k-1}\right)\right)
$$

DAMAS was introduced and is usually used with cyclic updates (that is, $i_{k}=k \bmod N$ ). This version will be called cyclic $D A M A S$ in the remainder of the paper. Random DAMAS, where the indices are chosen randomly, will be useful to obtain convergence guarantees. "DAMAS algorithm" will refer to both versions. The algorithm is usually initialized with $p_{i}^{0}=0$ for all $i$, and stopped when successive iterates are considered close enough. We emphasize that here, an iteration corresponds to the update of a unique coefficient, not to a full cycle.
In settings where the measurement noise cannot be neglected, the technique of diagonal removal can be used, where the diagonal of $\mathbf{C}$ is set to zero, and the matrix $\mathbf{A}$ is replaced by $\mathbf{A}_{\mathrm{dr}}$ with

$$
A_{\mathrm{dr}, i j}=\mathbf{d}_{i}^{H}\left(\mathbf{d}_{j} \mathbf{d}_{i}^{H}-\operatorname{diag}\left(\mathbf{d}_{j} \mathbf{d}_{i}^{H}\right)\right) \mathbf{d}_{j}^{H}
$$

where the operator $\operatorname{diag}(\mathbf{M})$ returns the diagonal matrix with diagonal taken from $\mathbf{M}$.

Remark 3. With the normalization of the steering vectors $\mathbf{d}_{j}$ used for the estimation of the powers with beamforming, the matrix $\mathbf{A}$ is replaced by $\tilde{\mathbf{A}}$ with values $\tilde{A}_{i j}=A_{i j} /\left\|\mathbf{d}_{i}\right\|_{2}^{4}$, which has values 1 on its diagonal. This formulation, used in the original introduction of the DAMAS algorithm, has the disadvantage that the matrix $\tilde{\mathbf{A}}$ is not symmetric semi-positive, and will not be used for our analysis of the DAMAS algorithm. Indeed, symmetry and positive semi-definiteness of $\mathbf{A}$ will be used in the proof of Theorem 2. Additionally, while the DAMAS algorithm considers thresholded Gauss-Seidel iterations, we note that symmetry and positivity of a matrix ensure the convergence of Gauss-Seidel iterations (see Theorem 11.2.2 in [20]). Nevertheless, for practical computations, the choice of $\mathbf{A}$ or $\tilde{\mathbf{A}}$ has no influence on the result of the algorithm. Indeed, the divisions by $\left\|\mathbf{d}_{i}\right\|_{2}^{4}$ in the definitions of $\tilde{b}_{i}$ and $\tilde{A}_{i j}$ cancel one another in (11).

\section{Fixed points of DAMAS}

In the cases where the DAMAS algorithm converges towards an estimate $\mathbf{p}$ of the power of the sources, this vector is necessarily a fixed point, defined by the following:

Definition 1. A fixed point $\mathbf{p}^{\star}$ of the DAMAS algorithm is defined by :

$$
p_{i}^{\star}=\max \left(0, \frac{1}{A_{i i}}\left(b_{i}-\sum_{l=0}^{i-1} A_{i l} p_{l}^{\star}-\sum_{l=i+1}^{N} A_{i l} p_{l}^{\star}\right)\right) .
$$

In this section, we will give characterizations of fixed points of the DAMAS algorithm as solutions to three convex minimization problems.

\subsection{Quadratic reformulation}

Our first step is the following result:

Proposition 1. A vector $\mathbf{p}^{\star}$ is a fixed point of the DAMAS algorithm if and only if there exist auxiliary variables $\lambda_{i}$ such that

$$
\left\{\begin{aligned}
p_{i}^{\star} & \geq 0 \\
p_{i}^{\star} & =\frac{1}{A_{i i}}\left(b_{i}-\sum_{l=0}^{i-1} A_{i l} p_{l}^{\star}-\sum_{l=i+1}^{N} A_{i l} p_{l}^{\star}\right)+\lambda_{i} \\
\lambda_{i} & \geq 0 \\
\lambda_{i} p_{i}^{\star} & =0
\end{aligned}\right.
$$

Proof. See Appendix A.1.

By interpreting this result as Karush-Kuhn-Tucker conditions for a convex optimization problem, the following characterization of a fixed point $\mathbf{p}^{\star}$ can be given: 
Theorem 1. A vector $\mathbf{p}^{\star}$ is a fixed point of the DAMAS algorithm if and only if it is a solution to the optimization problem

$$
\underset{\mathbf{p} \in \mathbf{R}_{+}^{N}}{\operatorname{argmin}} J(\mathbf{p})
$$

where

$$
J(\mathbf{p})=\frac{1}{2} \mathbf{p}^{T} \mathbf{A p}-\mathbf{b}^{T} \mathbf{p}
$$

Moreover, if $\mathbf{A}^{\star}$ (defined as the principal submatrix of $\mathbf{A}$ for the coordinates where the gradient $\nabla J\left(\mathbf{p}^{\star}\right)=\mathbf{A} \mathbf{p}^{\star}-\mathbf{b}$ is zero) is positive definite, then $\mathbf{p}^{\star}$ is unique.

Proof. See Appendix A.2

Remark 4. In the case where $\mathbf{A}$ is non-singular, the unconstrained global minimum of $J$ is $\mathbf{A}^{-1} \mathbf{b}$.

Fixed points can thus be found as minimizers of a constrained quadratic optimization problem. As will be shown, this problem can be solved by standard algorithms.

\subsection{Covariance matrix fitting problem}

The optimization problem (15) can be reformulated in a more meaningful way.

Theorem 2. Let $\mathbf{P}^{\star}$ be a solution to the problem

$$
\underset{\mathbf{P} \in \mathcal{D}_{+}^{N}}{\operatorname{argmin}}\left\|\mathbf{D P D} \mathbf{D}^{H}-\mathbf{C}\right\|_{F}^{2}
$$

where $\mathcal{D}_{+}^{N}$ is the set of $N \times N$ diagonal matrices with nonnegative coefficients, $\|\cdot\|_{F}$ is the Frobenius norm, and $\mathbf{D}$ is the matrix with columns $\mathbf{d}_{i}$.

Then $\mathbf{P}^{\star}$ is a solution of (17) if and only if its diagonal coefficients $\mathbf{p}^{\star}$ are solutions of problem (15).

Proof. See Appendix A.3.

In this formulation, the covariance matrix of the array measurements is directly taken as the input of the problem, and the beamforming step is unnecessary. This result unifies two different approaches for source localization, namely

- the DAMAS approach, where a beamforming map is first computed, and deconvolution (or, more generally inversion) of this beamforming map is achieved to yield source power estimations,

- the Covariance Matrix Fitting (CMF) approach, where estimated source powers are obtained by fitting source powers to the covariance matrix of the measurements, as introduced by Yardibi et al.[9].

A similar equivalence was proven in [21], where the authors show that, in an ideal setting where the spatial covariance matrix is perfectly known in an open, continuous domain, the infinite dimensional equations to be solved to match the unknown source power distribution to the beamforming map, or to the spatial covariance matrix, are equivalent. This result does not apply in practice, where measurements are noisy and discrete, covariances are estimated, and the problems are frequently illconditioned. In this case, Theorems 1 and 2 show that estimating the distribution of sources from the beamforming map with the DAMAS algorithm, or from the covariance matrix using an algorithm to solve problem (17), is equivalent. We note that this equivalence is specific to the DAMAS algorithm and problem (17), and is not true for any pair of beamforming deconvolution method and covariance matrix fitting method.

\subsection{Non-negative least-squares}

The fixed points of the DAMAS algorithm are solutions to a third optimization problem, important for practical purposes.

Theorem 3. Problems (15) and (17) are equivalent to the following non-negative least-squares problem

$$
\underset{\mathbf{p} \in \mathbf{R}_{+}^{N}}{\operatorname{argmin}}\|\tilde{\mathbf{D}} \mathbf{p}-\mathbf{c}\|_{2}^{2}
$$

where $\tilde{\mathbf{D}}$ is a $M^{2} \times N$ matrix, with columns given by the vectorization of the matrices $\mathbf{d}_{i} \mathbf{d}_{i}^{H}$, and $\mathbf{c}$ is a vectorized version of C.

Proof. See Appendix A.4.

Characterizing the power maps obtained by the DAMAS algorithm as solutions to a non-negative least-squares (NNLS) problem explains the fact that DAMAS yields sparse solutions, even if no sparsity prior is explicitly imposed on the solutions. Indeed, it is known that the NNLS problem behaves similarly to non-negative LASSO, i.e. yields sparse solutions (even without regularization) [22, 23].

As problem (17) is a non-negative least-squares fit of the spatial covariance matrix, and (18) a non-negative least-squares problem, the proposed method consisting of solving one of the equivalent problems (15), (17) or (18), will be called CMFNNLS.

Remark 5. We emphasize that problem (18) is different to the DAMAS-NNLS problem

$$
\underset{\mathbf{p} \in \mathbf{R}_{+}^{N}}{\operatorname{argmin}}\|\mathbf{A p}-\mathbf{b}\|_{2}^{2}
$$

introduced in [24], or equivalently

$$
\underset{\mathbf{p} \in \mathbf{R}_{+}^{N}}{\operatorname{argmin}} \frac{1}{2} \mathbf{p}^{T} \mathbf{A}^{2} \mathbf{p}-\mathbf{b}^{T} \mathbf{A p},
$$

which takes the beamforming map as input.

\subsection{Convergence of the DAMAS algorithm}

Finally, we conclude our theoretical analysis by a proof of convergence of the random DAMAS algorithm.

Theorem 4. Let $\mathbf{p}^{k}$ be the iterates of the random DAMAS algorithm. Then for $\varepsilon>0$ and $0<\rho<1$, and

$$
k \geq \frac{C_{1}}{\varepsilon}(1-\log \rho)+C_{2}
$$


where $C_{1}$ and $C_{2}$ are independent of $k, \varepsilon$ and $\rho$, the probability that $J\left(\mathbf{p}^{k}\right)-J\left(\mathbf{p}^{\star}\right) \leq \varepsilon$ is larger that $1-\rho$.

Moreover, under the condition of uniqueness of theorem 1 and with same probability,

$$
\left\|\mathbf{p}^{k}-\mathbf{p}^{\star}\right\|_{2} \leq C_{3} \varepsilon+C_{4} \sqrt{\varepsilon}
$$

with $C_{3}$ and $C_{4}$ independent of $k, \varepsilon$ and $\rho$.

Proof. See Appendix A.5.

\subsection{Diagonal terms}

When diagonal terms in $\mathbf{C}$ generated by uncorrelated measurement noises at each microphone cannot be neglected, diagonal removal can be applied to problem (17) by neglecting diagonal terms in the computation of the Frobenius norm. This problem is still convex, and its Hessian matrix is $\mathbf{A}_{\mathrm{dr}}$ defined in (12), which is as such semi-definite positive.

Equivalently, the diagonal can be accounted for by considering the modified problem

$$
\underset{\mathbf{P} \in \mathcal{D}_{+}^{N}, \mathbf{S} \in \mathcal{D}_{+}^{M}}{\operatorname{argmin}}\left\|\mathbf{D P} \mathbf{D}^{H}+\mathbf{S}-\mathbf{C}\right\|_{F}^{2} .
$$

where the diagonal of $\mathbf{S}$ is formed by the estimated variances of the noise at each sensor. This problem can be reformulated as a special case of problem (17):

$$
\underset{\overline{\mathbf{P}} \in \mathcal{D}_{+}^{N+M}}{\operatorname{argmin}}\left\|\overline{\mathbf{D}} \overline{\mathbf{P}} \overline{\mathbf{D}}^{H}-\mathbf{C}\right\|_{F}^{2} .
$$

where $\overline{\mathbf{D}}$ is the concatenation of $\mathbf{D}$ and the $M \times M$ identity matrix, and the diagonal of $\overline{\mathbf{P}}$ is the concatenation of the powers of the sources and the powers of the noise at each sensor.

\section{Optimization algorithms and numerical implementation}

The above results are not only useful to characterize and explain the properties of the power maps obtained by the DAMAS algorithm. As the fixed points of the DAMAS algorithm are shown to be solutions to standard optimization problems, the vast literature on convex optimization can be invoked for faster computations.

In addition to the cyclic and random DAMAS algorithms, we consider three algorithms to solve the CMF-NNLS problem (17). Each of these algorithms is based on one of the equivalent formulations (15), (17) and (18), and under the condition of unicity of Theorem 1, converges towards the same solution. A summary of the algorithms is given in Table 1.

Cyclic and random DAMAS algorithms. Coordinate descent with cyclic or random choice of the updated coordinate by equation (11). Cyclic DAMAS is the original DAMAS algorithm [1]. For the numerical results, the algorithm is implemented as a Matlab mex-file. This algorithm requires the explicit knowledge of all coefficients of the $N \times N$ matrix $\mathbf{A}$, and takes as input the beamforming map $\mathbf{b}$.
Interior point algorithm, quadratic programming. Theorem 1 shows that fixed points are solutions to the problem (15), a standard constrained convex quadratic program for which several algorithms exist. In our numerical experiments, we used the MATLAB R2019b functions quadprog (with the interior point algorithm and default parameters), which takes as input the matrix $\mathbf{A}$ and the beamforming map $\mathbf{b}$.

Lawson-Hanson algorithm. By Theorem 3, fixed points of the DAMAS algorithm are also solutions to problem (18), non-negative least-squares, a particular case of a constrained quadratic problem. This problem can be solved with the Lawson-Hanson algorithm [15], given in Appendix B. In this simple formulation, the algorithm takes as input the matrix $\tilde{\mathbf{D}}$ and the SCM $\mathbf{C}$ as the vector $\mathbf{c}$. We used here the MATLAB R2019b implementation (lsqnonneg) of the Lawson-Hanson algorithm.

Optimized Lawson-Hanson algorithm. Finally, by using the formulation (17) given by Theorem 2 and the structure of $\mathbf{A}$ given by eq. (10), a faster implementation of the LawsonHanson algorithm can be obtained. Details are given in Appendix $\mathrm{B}$.

The improvement in time and memory obtained by using the structure of the problem can be seen by considering the storage cost of $\mathbf{A}$, and the computational complexity of a product of $\mathbf{A}$ with a vector. Although $\mathbf{A}$ does not appear explicitly in problems (17) and (18), it is involved in the computations of the Lawson-Hanson algorithm, and dominates its numerical complexity. Orders of magnitudes given in table 2 show that using the structure of $\mathbf{A}$ (see eq. (10)) offers a substantial improvement in both memory and time, as the storage space and computational complexity are linear with respect to the size of the discretization $N$ instead of quadratic.

This approach is similar to the use of a Fast Fourier Transform algorithm instead of the explicit Discrete Fourier Transform matrix, with computational complexity reduced to $O(N \log N)$ from $O\left(N^{2}\right)$, and is not specific to the LH algorithm. However, algorithms that need explicit knowledge of the coefficients of A (e.g., the DAMAS algorithm), cannot profit from this factorization.

The cost of a DAMAS iteration (i.e. update of one coefficient) is also given, using the explicit knowledge of $\mathbf{A}$ if memory is sufficient to hold the entire matrix $(O(M))$, and by recomputing necessary values of $\mathbf{A}$ from $\mathbf{D}$ when needed for lower memory usage $(O(N M))$.

In addition, the Lawson-Hanson algorithm will also be used to solve the DAMAS-NNLS problem (20), using the same optimization for the product by $\mathbf{A}$.

\section{Experimental results}

The methods are tested experimentally upon signals recorded in an anechoic chamber. The setup of the experiments is identical to the experiments described in [25]. 


\begin{tabular}{cccc} 
Name & Implementation & 2D & 3D \\
\hline Cyclic DAMAS & mex & $682 \mathrm{~s}$ & - \\
Random DAMAS & mex & $277 \mathrm{~s}$ & - \\
Interior point & quadprog & $153 \mathrm{~s}$ & - \\
Lawson-Hanson & Alg. 1, lsqnonneg & $10.6 \mathrm{~s}$ & - \\
Lawson-Hanson & Alg. 1 using (10) & $1.5 \mathrm{~s}$ & $7 \mathrm{~min}$ \\
\hline DAMAS-NNLS, LH & Alg. 1 using (10) & $8.1 \mathrm{~s}$ & $47 \mathrm{~min}$ \\
\hline
\end{tabular}

Table 1: Optimization algorithms. The first five algorithms solve the equivalent CMF-NNLS problems (15), (17) and (18). The last algorithm solves the DAMAS-NNLS problem (19). Algorithm 1 is given in Appendix B

\begin{tabular}{cccc} 
& Memory & Product, LH iteration & DAMAS iteratio \\
\hline Naive & $O\left(N^{2}\right)$ & $O\left(N^{2}\right)$ & $O(N)$ \\
\hline Optimized & $O(N M)$ & $O\left(N M^{2}\right)$ & $O(N M)$ \\
\hline
\end{tabular}

Table 2: Storage cost and matrix-vector product time complexity for the matrix A with and without using the structure given by eq. (10). In large scale applications, the size of the grid $N$ is larger than the number of microphones squared $M^{2}$.

Sources built out of baffled broadband omnidirectional loudspeakers (Visaton-BF32 - [150Hz-20kHz]) are used. The implemented acoustic array uses MEMS digital microphones (Invensense - INMP441) with a $26 \mathrm{dBFS}$ sensitivity $(1 \mathrm{kHz}, 94 \mathrm{~dB}-$ SPL) and a flat response in the band $[150 \mathrm{~Hz}-15 \mathrm{kHz}]$.

The antenna counts 128 elements distributed along 16 linear rays. On each ray, the 8 microphones are spaced regularly at $17 \mathrm{~cm}$ intervals, the distance between the first microphone and the center of the array follows a pseudo-random distribution.

The microphone array and the sources are pictured on fig. 1 and 2-(a). They are located in two parallel planes, at a distance $d=4.4 \mathrm{~m}$.

The microphone signals are sampled at $F_{s}=50 \mathrm{kHz}$ and analyzed by Short-Term Fourier Transform, with a 2048 samples Hann window (41ms duration and $75 \%$ overlap).

Data are processed using Matlab 2019b on a personal laptop equipped with an Intel Core i7-7820HQ @ 2.90GHz × $8 \mathrm{CPU}$ and 16 GB memory.

\section{1. $2 D$ inversion, comparison of the algorithms}

We first compare the algorithms in a medium-dimensional case, at frequency $F=1806 \mathrm{~Hz}$. The search space is discretized using a $N=180 \times 60=10800$ grid of size $3 \mathrm{~m} \times 1 \mathrm{~m}$ at a distance of $4.4 \mathrm{~m}$ to the microphone array. Here, only the $M=64$ central microphones are used. This choice was made to lower the intrinsic resolution of the array and match the performances of "off the shelf" commercial systems. The output of the beamformer is given on figure 2-(b). The sources cannot be resolved by beamforming.

The two versions of DAMAS (cyclic and random), the two version of the Lawson-Hanson algorithm (naive and optimized) and the interior-point algorithm are tested. The LH algorithm converges in a finite number of iterations with a positive definite matrix $\mathbf{A}^{\star}$, thus providing the exact unique minimizer $\mathbf{p}^{\star}$ of (15). DAMAS and the interior point algorithm are run until the

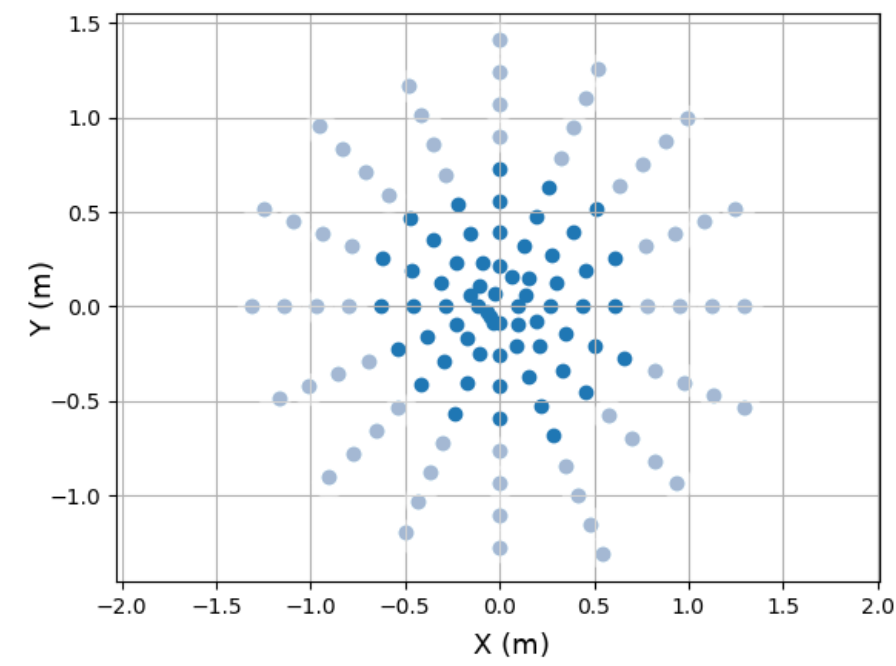

Figure 1: Microphone array. Microphones represented by lighter dots were not used in the $2 \mathrm{D}$ experiments.

relative gap

$$
\delta_{k}=\frac{\left\|\tilde{\mathbf{D}} \mathbf{p}^{k}-\mathbf{c}\right\|_{2}^{2}-\left\|\tilde{\mathbf{D}} \mathbf{p}^{\star}-\mathbf{c}\right\|_{2}^{2}}{\left\|\tilde{\mathbf{D}} \mathbf{p}^{\star}-\mathbf{c}\right\|_{2}^{2}}
$$

reaches $10^{-4}$. Running times are reported in Table 1 .

The results of the random DAMAS algorithm are given on figure 2-(c) (cyclic DAMAS yields similar results). At the chosen stopping criterion, DAMAS cannot fully separate the two closely spaced source. Results of the CMF-NNLS method with the Lawson-Hanson algorithm are given on figure 2-(d) (both versions yield identical results, and quadprog yields similar results). The four sources are clearly separated. The difference in performances are entirely caused by stopping the DAMAS algorithm. Indeed, at least for random DAMAS, Theorem 4 shows that running the algorithm for a sufficiently large number of iterations would yield results arbitrarily close to the actual minimizer $\mathbf{p}^{\star}$, with probability as close to one as needed.

The five algorithms are compared by plotting $\delta_{k}$ in function of the computational time on figure 3 , with varying number of iterations. The gap in computational speed between the two versions of the LH algorithm is entirely explained by the use of the factorization (10). Decrease of the objective function for DAMAS is consistent with Theorem 4.

Results of the DAMAS-NNLS method are similar but slightly different to CMF-NNLS (figure 2-(e)), which is expected as they consider different optimization problems. The longer running time is expected as the objective function of the DAMAS-NNLS and its gradient involves more products by $\mathbf{A}$ than that of CMF-NNLS.

In the remainder of the article, the optimized LawsonHanson algorithm will be used to solve the CMF-NNLS problem. 


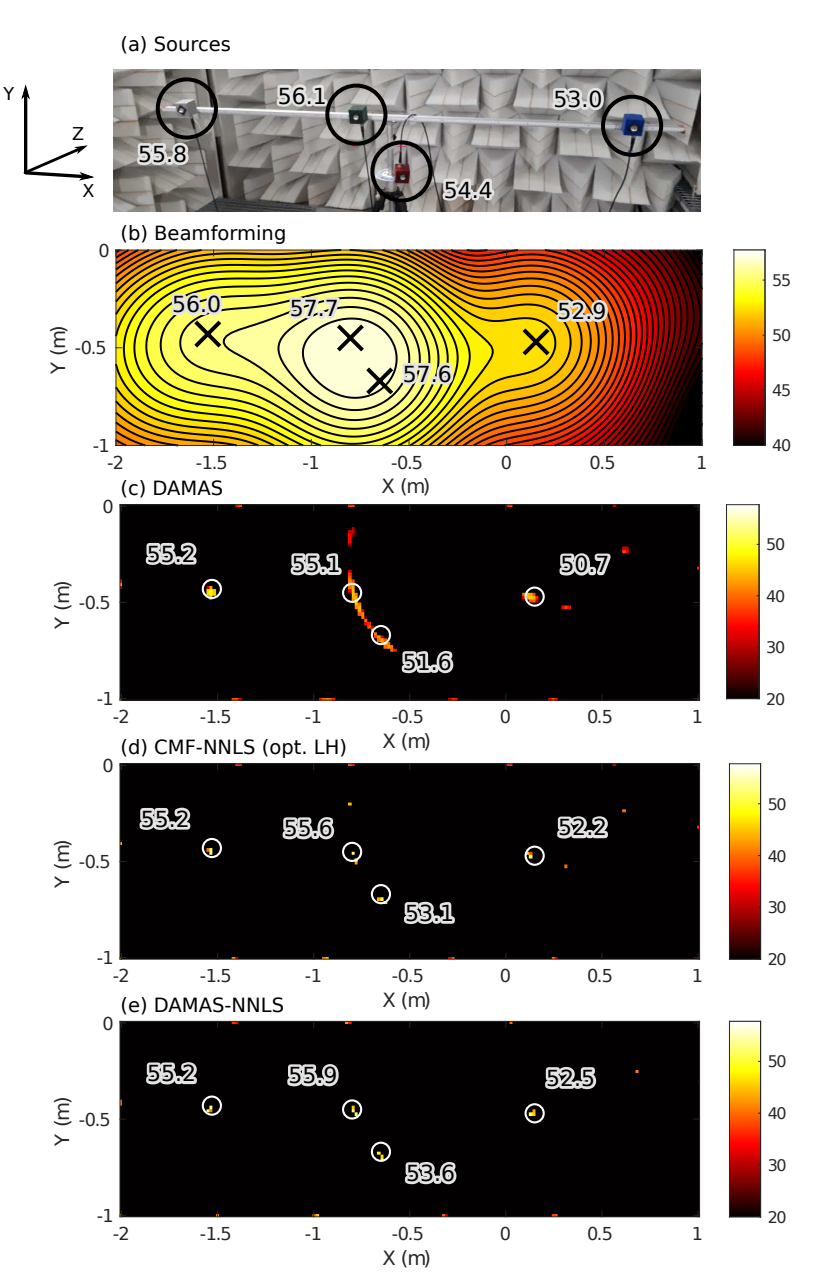

Figure 2: 2D experiment, $F=1806 \mathrm{~Hz}$. Comparison of (a) actual powers of the sources, (b) beamforming, (c) DAMAS, (d) CMF-NNLS, and (e) DAMASNNLS . Powers in dB. Actual positions are indicated by crosses in (b), circles in (c) (d) and (e). Numbers indicate the power summed in the circles of radius $0.05 \mathrm{~cm}$ around the true locations of the sources.

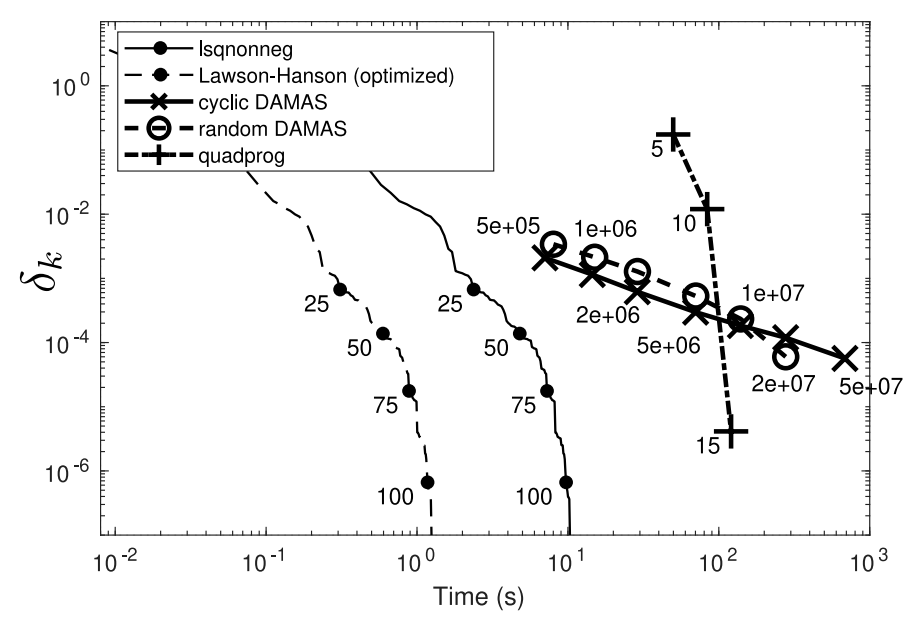

Figure 3: Decay of the gap (25) with respect to computational time. The number of iterations is indicated with labels.
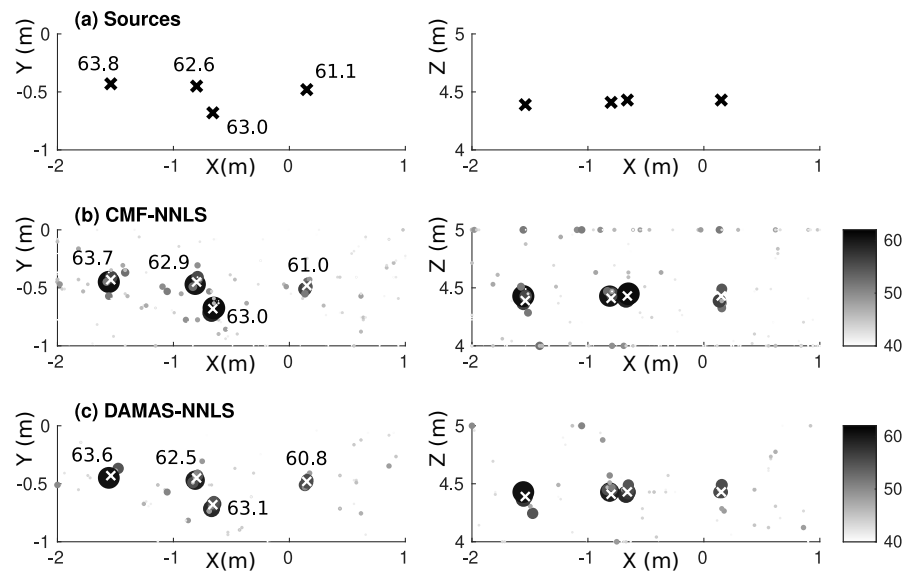

Figure 4: 3D experiment, $F=4858 \mathrm{~Hz}$. Front (left) and top (right) view. (a) Positions and powers of the sources. Results of (b) CMF-NNLS and (c) DAMAS-NNLS. Estimated powers and positions of the sources are indicated by discs with radius proportional to the power, actual positions by crosses. Indicated powers in $\mathrm{dB}$ are obtained by summing the estimated powers in a sphere of radius $R=0.08 \mathrm{~m}$ around the actual position of the sources.

\subsection{D problem, large scale inversion}

The numerical efficiency of the optimized Lawson-Hanson algorithm for solving the CMF-NNLS problem allows application to large scale problems. Here, the search space is extended to a $3 \mathrm{D}$ region of size $3 \mathrm{~m} \times 1 \mathrm{~m} \times 1 \mathrm{~m}$, discretized over $N=150 \times 50 \times 50=3.75 \times 10^{5}$ points (to our best knowledge, the size of this problem is 30 times the size of the largest problem previously considered with DAMAS in the literature [3]). Positions and powers of the sources are given on Figure 4-(a). All $M=128$ microphones are used, and $F=4858 \mathrm{~Hz}$. Results of CMF-NNLS are given on Figure 4-(b). Computation time for this result is approximately $7 \mathrm{~min}$. The four sources are easily identifiable and they are located in the same plane, in coherence with the experimental setup where the sources were located in a plane parallel to the array. Low power spurious sources appear on the boundary of the domain, likely because of a mismatch between the model and the experimental conditions (wave velocity, reflections on the equipment, etc.). DAMAS-NNLS converges in $47 \mathrm{~min}$, with similar powers and less spurious sources (Figure 4-(c)).

The DAMAS algorithm, and more generally algorithms that need explicit knowledge of the coefficients of $\mathbf{A}$, cannot be applied in this case with the equipment used here. Indeed, the size of the matrix A scales as $O\left(N^{2}\right)$, which here would necessitates prohibitive memory space (here, approximately $1 \mathrm{~TB}$ as double floats). Alternatively, the structure of $\mathbf{A}$ can be used to decrease the memory requirements, but at a cost of a computational complexity multiplied by $M$ (see Table 2 ), which prevents to obtain usable results in a reasonable time.

\section{Performance analysis}

Simulations are now used to assess the performances of the original DAMAS algorithm, CMF-NNLS and DAMAS-NNLS formulation of the problem with the optimized LH algorithm. 
Three sources with powers $p_{1}=83.0 \mathrm{~dB}$ and $p_{2}=$ $89.0 \mathrm{~dB}$ and $p_{3}=76.0 \mathrm{~dB}$ at coordinates $(0.60,0.50,4.4)$, $(0.67,0.67,4.4)$ and $(0.25,0.45,4.4)$ respectively are simulated in a similar setting as in the $2 \mathrm{D}$ experiments, at $F=4858 \mathrm{~Hz}$. The region of interest has dimensions $1 \mathrm{~m} \times 1 \mathrm{~m}$, discretized over a grid of $N=100 \times 100=10^{4}$ points. Measurements at the sensors are noisy, with a signal to noise ratio of $7.83 \mathrm{~dB}$ for the first 32 sensors and $1.83 \mathrm{~dB}$ for the last 32 sensors, with a global SNR of $3.88 \mathrm{~dB}$. 500 snapshots are used to compute the sample covariance matrix of the measurements.

Source power maps obtained by beamforming, DAMAS, CMF-NNLS and DAMAS-NNLS are pictured in figure 5. $10^{7}$ DAMAS iterations are used, with an average computation time of $143 \mathrm{~s}$. Average computation times are 1.29s for CMF-NNLS and 5.1s for DAMAS-NNLS. All methods use diagonal removal.

The wavelength is too large to allow beamforming to separate the sources. Power maps obtained by CMF-NNLS and DAMAS-NNLS are sparser than the power maps returned by DAMAS.

200 realizations of the numerical experiments are used to assess the performances of the three methods. As in [5], values of the powers of the sources are taken by summing the estimated powers around the actual position of the source, here on the position of the source and its eight neighbouring nodes. Histograms of the estimation errors of the powers of the 3 sources $\left(\Delta_{1}, \Delta_{2}\right.$ and $\Delta_{3}$, Specific level error in [5], computed as the difference between the actual and estimated powers, both in $\mathrm{dB}$ ) are given for the four methods. In addition, histograms of the negative sum of the estimated powers outside of the neighborhood of the three sources $\left(\Delta_{0}\right.$, Inverse level error in [5], in $\left.\mathrm{dB}\right)$ are given.

Comparing DAMAS and CMF-NNLS, DAMAS powers are underestimated. This bias is caused by the spread of the sources at $10^{7}$ DAMAS iterations compared to the result of the LawsonHanson algorithm, which is the actual solution of the CMFNNLS problem. DAMAS-NNLS yields slightly better results than CMF-NNLS, at a cost of a higher computational time.

\section{Conclusion}

Since its introduction in 2006, the DAMAS algorithm has been largely used in complement to the classical beamforming technique to identify the accurate location and power of acoustic sources. However this robust method essentially based on empirical considerations, implements basic numerical processes and therefore suffers from a high demand in memory space and computational time which prevents to solve large scale problems. In this work, we investigated the theoretical foundations of the DAMAS algorithm.

We have shown that fixed points (i.e. possible limits) of the DAMAS algorithm are solutions to a non-negative least-squares problem, or, alternatively, least-squares fit of a diagonal covariance matrix to the data. We also have shown that the beamforming step, starting point of DAMAS is redundant and can be avoided. Additionnaly, it was shown that under a condition
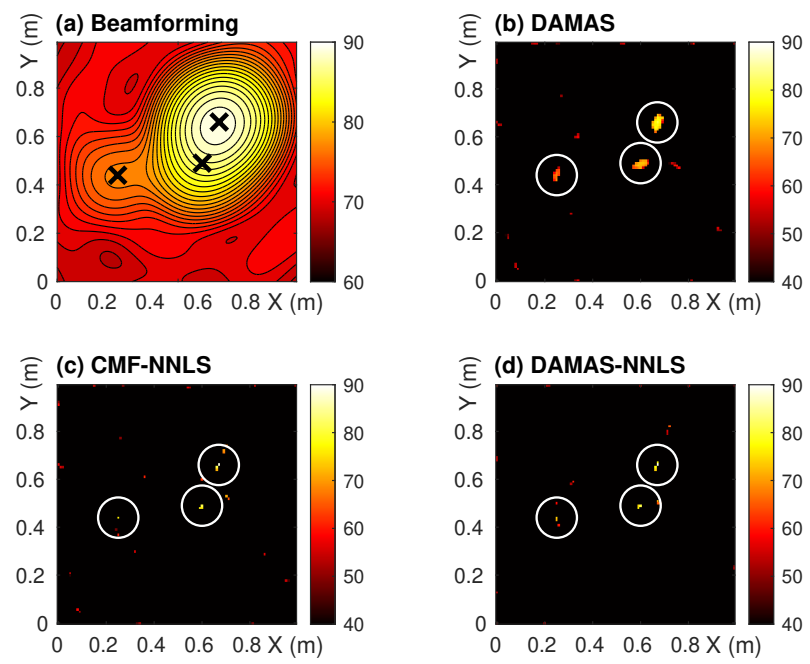

Figure 5: 2D simulations, $f=4858 \mathrm{~Hz}$. Results of (a) beamforming, (b) DAMAS algorithm, (c) CMF-NNLS, (d) DAMAS-NNLS. Powers in dB.
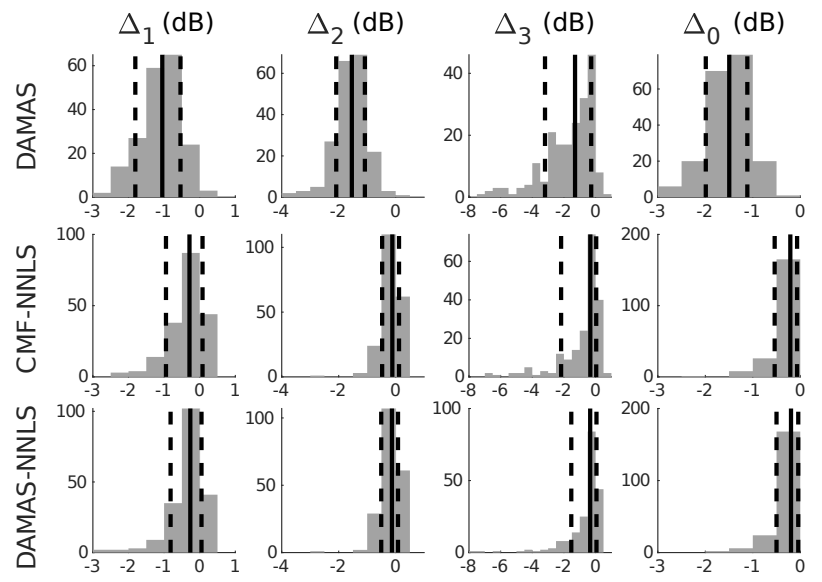

Figure 6: Histograms of the estimation errors (in $\mathrm{dB}$ ) for DAMAS, CMFNNLS, DAMAS-NNLS: $\Delta_{1}, \Delta_{2}, \Delta_{3}$ specific level errors for the three sources, $\Delta_{0}$ inverse level error, as defined in [5]. Sample size: 200. Solid line indicates the median error, dashed lines the 14th and 86th percentile. 
that can be checked at convergence, the solution of the nonnegative least squares problem is unique, and in that case, that the DAMAS algorithm converges towards this solution.

To reduce the computation complexity, it was shown that the specific structure of the beamforming kernel A could be used to accelerate matrix-vector products involved in the LawsonHanson algorithm. This approach allows to use wide spread algorithms dedicated to the resolution of classical optimization problems and far more efficient than the standard iterative deconvolution process. This improved efficiency allowing applications to large-scale problems has been implemented to process experimental data and successfully locate four sources in a $3 \mathrm{D}$ region. In complement, a numerical study was led to investigate the ability of this approach to provide an accurate measurement of the power of the sources.

The gain in computational efficiency were obtained under the assumption that the size of the discretization of the space was larger than the size of the spatial covariance matrix. The case of larger microphone arrays will likely necessitates specific numerical methods.

\section{Appendix A. Proofs}

\section{Appendix A.1. Proof of Proposition 1}

We first prove that $(13) \Rightarrow$ (14). We first consider the case where $p_{i}^{\star}>0$. Then by setting $\lambda_{i}=0$, the four equations of (14) are satisfied. In the case $p_{i}^{\star}=0$, we set $\lambda_{i}=$ $-\frac{1}{A_{i i}}\left(b_{i}-\sum_{l=0}^{i-1} A_{i l} p_{l}^{\star}-\sum_{l=i+1}^{N} A_{i l} p_{l}^{\star}\right) \geq 0$.

To prove that (14) $\Rightarrow$ (13), we proceed similarly. If $\lambda_{i}=0$, then (13) is true for $p_{i}^{\star}$. Alternatively, if $\lambda_{i}>0$, then $p_{i}^{\star}=0$ and $\frac{1}{A_{i i}}\left(b_{i}-\sum_{l=0}^{i-1} A_{i l} p_{l}^{\star}-\sum_{l=i+1}^{N} A_{i l} p_{l}^{\star}\right)<0$ which implies (13) for $p_{i}^{\star}$.

\section{Appendix A.2. Proof of Theorem 1}

As $J$ is a convex function ( $\mathbf{A}$ is semi-definite positive) and $\mathbf{R}_{+}^{N}$ a convex domain, global minima of the optimization problem (15) are characterized by the Karush-Kuhn-Tucker (KKT) conditions [14], which here write:

$$
\left\{\begin{aligned}
\nabla J\left(\mathbf{p}^{\star}\right)-\mu & =0 \\
p_{i}^{\star} & \geq 0 \\
\mu_{i} & \geq 0 \\
\mu_{i} p_{i}^{\star} & =0
\end{aligned}\right.
$$

where $\mu=\left(\mu_{1}, \ldots, \mu_{n}\right)$ is a vector of KKT multipliers.

Expliciting the first equation using $\nabla J(\mathbf{p})=\mathbf{A p}-\mathbf{b}$, we get

$$
\sum_{l=1}^{N} A_{i l} p_{l}^{\star}-b_{i}-\mu_{i}=0
$$

or, equivalently,

$$
p_{i}^{\star}=\frac{1}{A_{i i}}\left(b_{i}-\sum_{l=0}^{i-1} A_{i l} p_{l}^{\star}-\sum_{l=i+1}^{N} A_{i l} p_{l}^{\star}\right)+\frac{\mu_{i}}{A_{i i}}
$$

Identifying $\mu_{i} / A_{i i}$ as $\lambda_{i}$, the KKT conditions (A.1) are equivalent to the characterization (14) of fixed points of the DAMAS algorithm.

We now give a sufficient condition for uniqueness of $\mathbf{p}^{\star}$. We denote $S$ the set of coordinates where $\mu$ is zero, and the restriction of a vector $\mathbf{x}$ to this set $\mathbf{x}_{S}$, and likewise for its complement $\bar{S}$, assumed nonempty. We have $\mathbf{p}_{\bar{S}}^{\star}=0, \mu_{\bar{S}} \geq 0$ and $\nabla J\left(\mathbf{p}^{\star}\right)=\mu$ from the KKT conditions. As $J$ is a quadratic function, for any $\mathbf{p}$ and $\mathbf{h}$,

$$
J(\mathbf{p}+\mathbf{h})-J(\mathbf{p})=\langle\nabla J(\mathbf{p}), \mathbf{h}\rangle+\frac{1}{2} \mathbf{h}^{H} \mathbf{A} \mathbf{h} .
$$

With $\mathbf{h}$ such that $\mathbf{p}^{\star}+\mathbf{h}$ is feasible (which implies that $\mathbf{h}_{\bar{S}} \geq 0$ ),

$$
J\left(\mathbf{p}^{\star}+\mathbf{h}\right)-J\left(\mathbf{p}^{\star}\right)=\left\langle\mu_{\bar{S}}, \mathbf{h}_{\bar{S}}\right\rangle+\left\langle\mu_{S}, \mathbf{h}_{S}\right\rangle+\frac{1}{2} \mathbf{h}^{H} \mathbf{A} \mathbf{h}
$$

In the right hand side of (A.5), the first term is nonnegative, the second zero, and the third nonnegative (stemming from the semi-definite positivity of $\mathbf{A}$ ).

We then have the two inequalities

$$
\begin{aligned}
& J\left(\mathbf{p}^{\star}+\mathbf{h}\right)-J\left(\mathbf{p}^{\star}\right) \geq\left\langle\mu_{\bar{S}}, \mathbf{h}_{\bar{S}}\right\rangle \geq \min _{i \in \bar{S}} \mu_{i}\left\|\mathbf{h}_{\bar{S}}\right\|_{1} \\
& J\left(\mathbf{p}^{\star}+\mathbf{h}\right)-J\left(\mathbf{p}^{\star}\right) \geq \frac{1}{2} \mathbf{h}^{H} \mathbf{A} \mathbf{h} .
\end{aligned}
$$

If $\mathbf{p}^{\star}+\mathbf{h}$ is a minimizer, the left hand side of the above equations is zero, implying $\left\|\mathbf{h}_{\bar{S}}\right\|_{1}=0$ and $\mathbf{h}_{\bar{S}}=0$. Combining with (A.7) yields $0=\frac{1}{2} \mathbf{h}^{H} \mathbf{A} \mathbf{h}=\frac{1}{2} \mathbf{h}_{S}^{H} \mathbf{A}^{\star} \mathbf{h}_{S}$. If $\mathbf{A}^{\star}$ is positive definite, this implies $\mathbf{h}_{S}=0$. Combining with $\mathbf{h}_{\bar{S}}=0$ implies $\mathbf{h}=0$ and unicity of the minimizer. The same reasoning can be applied when $\bar{S}$ is empty and $\mathbf{A}^{\star}=\mathbf{A}$.

\section{Appendix A.3. Proof of Theorem 2}

Since $\mathbf{P}$ is diagonal, we first observe that with $p_{i}$ the diagonal coefficients of $\mathbf{P}, \mathbf{D P D}{ }^{H}=\sum_{i} p_{i} \mathbf{d}_{i} \mathbf{d}_{i}^{H}$. Then,

$$
\begin{aligned}
\left\|\mathbf{D P D} \mathbf{D}^{H}-\mathbf{C}\right\|_{F}^{2} & =\operatorname{tr}\left(\left(\mathbf{D P D} \mathbf{D}^{H}-\mathbf{C}\right)^{H}\left(\mathbf{D P D} \mathbf{D}^{H}-\mathbf{C}\right)\right) \\
& =\operatorname{tr}\left(\left(\sum_{i=1}^{N} p_{i} \mathbf{d}_{i} \mathbf{d}_{i}^{H}-\mathbf{C}\right)^{H}\left(\sum_{j=1}^{N} p_{j} \mathbf{d}_{j} d d_{j}^{H}-\mathbf{C}\right)\right) \\
& =\sum_{i=1}^{N} \sum_{j=1}^{N} p_{i} p_{j} \operatorname{tr}\left(\mathbf{d}_{i} \mathbf{d}_{i}^{H} \mathbf{d}_{j} \mathbf{d}_{j}^{H}\right) \\
& =\sum_{i=1}^{N} \sum_{j=1}^{N} p_{i} p_{j}\left|\mathbf{d}_{i}^{H} \mathbf{d}_{j}\right|^{2}-2 \sum_{i=1}^{N} p_{i} \mathbf{d}_{i}^{H} \mathbf{C r}\left(\mathbf{d}_{i} \mathbf{d}_{i}^{H} \mathbf{C}\right)+\operatorname{tr}\left(\mathbf{C}^{2}\right) \\
& =2 J(\mathbf{p})+\operatorname{tr}\left(\mathbf{C}^{2}\right) .
\end{aligned}
$$

As the objective functions of (15) and (17) are related by an affine transformation with a strictly positive multiplicative coefficient, they share the same minimizers. 


\section{Appendix A.4. Proof of Theorem 3}

With $\tilde{\mathbf{d}}_{i}$ the columns of $\tilde{\mathbf{D}}$,

$$
\|\tilde{\mathbf{D}} \mathbf{p}-\mathbf{c}\|_{2}^{2}=\sum_{i=1}^{N} \sum_{j=1}^{N} p_{i} p_{j} \tilde{\mathbf{d}}_{i}^{H} \tilde{\mathbf{d}}_{j}-2 \sum_{i=1}^{N} p_{i} \tilde{\mathbf{d}}_{i}^{H} \mathbf{c}+\|\mathbf{c}\|_{2}^{2}
$$

Equality with (A.11) is obtained by remarking that

$$
\begin{aligned}
\tilde{\mathbf{d}}_{i}^{H} \tilde{\mathbf{d}}_{j} & =\operatorname{tr}\left(\left(\mathbf{d}_{i} \mathbf{d}_{i}^{H}\right)^{H}\left(\mathbf{d}_{j} \mathbf{d}_{j}^{H}\right)\right) \\
& =\left|\mathbf{d}_{i}^{H} \mathbf{d}_{j}\right|^{2} \\
\tilde{\mathbf{d}}_{i}^{H} \mathbf{c} & =\operatorname{tr}\left(\left(\mathbf{d}_{i} \mathbf{d}_{i}^{H}\right)^{H} \mathbf{C}\right) \\
& =\mathbf{d}_{i}^{H} \mathbf{C} \mathbf{d}_{i}
\end{aligned}
$$

\section{Appendix A.5. Proof of Theorem 4}

The random DAMAS algorithm can be interpreted as the Uniform Coordinate Descent for Composite Functions algorithm of [16], by reformulating problem (15) as the unconstrained problem

$$
\underset{\mathbf{p} \in \mathbf{R}^{N}}{\operatorname{argmin}} J(\mathbf{p})+\sum_{i=1}^{N} \Psi\left(p_{i}\right)
$$

where $\Psi\left(p_{i}\right)=0$ if $p_{i} \geq 0$, and $\Psi\left(p_{i}\right)=+\infty$ if $p_{i}<0$. The algorithm operates as follows:

1. Choose randomly an index $i_{k}$ with probability $1 / N$

2. $T^{k}=\operatorname{argmin}_{t} \nabla\left(J\left(\mathbf{p}^{k}\right)\right)_{i_{k}} t+\frac{A_{i_{k} i_{k}}}{2} t^{2}+\Psi\left(p_{i_{k}}^{k}+t\right)$

3. $p_{j}^{k+1}=p_{j}^{k}$ for $j \neq i_{k}$

4. $p_{i_{k}}^{k+1}=p_{i_{k}}^{k}+T^{k}$

Solution of step 2 is either

$$
T=\frac{1}{A_{i_{k} i_{k}}}\left(b_{i_{k}}-\sum_{j=1}^{N} A_{i_{k} j} p_{j}^{k}\right)
$$

if $T+p_{i_{k}}^{k} \geq 0$, or else $T^{k}=-p_{i_{k}}^{k}$. In either case, $p_{i_{k}}^{k+1}$ is given by the DAMAS iteration (11). Theorem 5 of [16] yields (21).

We now extend the results of Appendix A.2 to give a bound on $\|\mathbf{h}\|_{2}$ in function of $\varepsilon=J\left(\mathbf{p}^{\star}+\mathbf{h}\right)-J\left(\mathbf{p}^{\star}\right)$, in cases where $\mathbf{A}^{\star}$ is positive definite with smallest eigenvalue $\kappa>0$, and $\bar{S}$ is non-empty.

Firstly, using (A.6), we get

$$
\left\|\mathbf{h}_{\bar{S}}\right\|_{1} \leq \frac{\varepsilon}{\min _{i \in \bar{S}} \mu_{i}}
$$

With $\mathbf{A}_{\bar{S}}$ the principal submatrix (and therefore, semi-definite positive) of $\mathbf{A}$ for indices in $\bar{S}$, and $\mathbf{A}_{\bar{S} S}$ the rectangular matrix for rows in $\bar{S}$ and columns in $S$,

$$
\mathbf{h}^{H} \mathbf{A} \mathbf{h}=\left(\mathbf{h}_{S}^{H} \mathbf{A}^{\star} \mathbf{h}_{S}+\mathbf{h}_{\bar{S}}^{H} \mathbf{A}_{\bar{S} S} \mathbf{h}_{S}+\mathbf{h}_{S}^{H} \mathbf{A}_{\bar{S} S}^{H} \mathbf{h}_{\bar{S}}+\mathbf{h}_{\bar{S}}^{H} \mathbf{A}_{\bar{S}} \mathbf{h}_{\bar{S}}\right) .
$$

As $\mathbf{h}_{S}^{H} \mathbf{A}^{\star} \mathbf{h}_{S} \geq \kappa\left\|\mathbf{h}_{S}\right\|_{2}^{2}, \mathbf{h}_{\bar{S}}^{H} \mathbf{A}_{\bar{S}} \mathbf{h}_{\bar{S}} \geq 0, \mathbf{h}^{H} \mathbf{A} \mathbf{h} \leq 2 \varepsilon$ from (A.7),

$$
\kappa|| \mathbf{h}_{S} \|_{2}^{2} \leq 2 \varepsilon+2\left|\mathbf{h}_{\bar{S}}^{H} \mathbf{A}_{\bar{S} S} \mathbf{h}_{S}\right|
$$

Using Cauchy-Schwarz inequality, $\left\|\mathbf{A}_{\bar{S} S}\right\|$ the operator norm of $\mathbf{A}_{\bar{S} S}$, i.e. such that $\left\|\mathbf{A}_{\bar{S} S} \mathbf{x}\right\|_{2} \leq\left\|\mathbf{A}_{\bar{S} S}\left|\|\mid \mathbf{x}\|_{2}\right.\right.$, the bound $\left\|\mathbf{h}_{\bar{S}}\right\|_{2} \leq$ $\left\|\mathbf{h}_{\bar{S}}\right\|_{1}$, and (A.20)

$$
\begin{aligned}
\left|\mathbf{h}_{\bar{S}}^{H} \mathbf{A}_{\bar{S} S} \mathbf{h}_{S}\right| & \leq\left\|\mathbf{h}_{\bar{S}}\right\|_{2}\left\|\mathbf{A}_{\bar{S} S} \mathbf{h}_{S}\right\|_{2} \\
& \leq\left\|\mathbf{h}_{\bar{S}}\right\|_{1}\left\|\mathbf{A}_{\bar{S} S}\right\|\left\|\mathbf{h}_{S}\right\|_{2} \\
& \leq \frac{\varepsilon}{\min _{i \in \bar{S}} \mu_{i}}\left\|\mathbf{A}_{\bar{S} S}\right\|\left\|\mathbf{h}_{S}\right\|_{2}
\end{aligned}
$$

Combining with (A.22) yields

$$
\kappa\left\|\mathbf{h}_{S}\right\|_{2}^{2}-\frac{2\left\|\mathbf{A}_{\bar{S} S}\right\| \varepsilon}{\min _{i \in \bar{S}} \mu_{i}}\left\|\mathbf{h}_{S}\right\|_{2}-2 \varepsilon \leq 0
$$

Solving this quadratic inequality for $\left\|\mathbf{h}_{S}\right\|_{2}$ yields

$$
\left\|\mathbf{h}_{S}\right\|_{2} \leq \frac{\left\|\mathbf{A}_{\bar{S} S}\right\| \varepsilon}{\kappa \min _{i \in \bar{S}} \mu_{i}}+\sqrt{\left(\frac{\left\|\mathbf{A}_{\bar{S} S}\right\| \varepsilon}{\kappa \min _{i \in \bar{S}} \mu_{i}}\right)^{2}+\frac{2 \varepsilon}{\kappa}}
$$

Using $a+\sqrt{a^{2}+b} \leq a+\sqrt{a^{2}}+\sqrt{b}=2 a+\sqrt{b}$ for $a, b \geq 0$ and combining with inequality (A.20) using the triangle inequality yields

$$
\|\mathbf{h}\|_{2} \leq \frac{1}{\min _{i \in \bar{S}} \mu_{i}}\left(1+\frac{2\left\|\mathbf{A}_{\bar{S} S}\right\|}{\kappa}\right) \varepsilon+\sqrt{\frac{2 \varepsilon}{\kappa}}
$$

In the case of empty $\bar{S}$, we recover the simple bound $\|\mathbf{h}\|_{2} \leq$ $\sqrt{2 \varepsilon / \kappa}$.

\section{Appendix B. Implementation details}

The Lawson-Hanson algorithm [15] for solving $\operatorname{argmin}_{\mathbf{x} \in \mathbf{R}_{+}^{N}}\|\mathbf{M x}-\mathbf{y}\|_{2}^{2}$ is given in Algorithm 1 (here, $\mathbf{M}_{P}$ denotes the restriction of $\mathbf{M}$ to the columns with indices in $P$, keeping all rows, $\bar{P}$ the set difference of $\{1, \ldots, N\}$ and $P$, and $\cdot \cdot^{\dagger}$ the Moore-Penrose pseudoinverse). It takes as input the matrix $\mathbf{M}$, the vector $\mathbf{y}$ and a tolerance $\tau$, to be set at 0 for an exact solution. Here, an iteration of the LH algorithm is considered to be an execution of the outer loop.

In the course of the algorithm, new indices are found by finding the maximal coefficient of $\mathbf{w}$. For problems (17) and (18), computation of $\mathbf{w}$ is equivalent to computing $\nabla J(\mathbf{p})=\mathbf{A p}-\mathbf{b}$, and dominates the computational complexity of the algorithm. With $\mathbf{y}=\mathbf{A x}, y_{i}$ is given by $y_{i}=\sum_{j=1}^{N} A_{i j} x_{j}$ for a cost of $O(N)$ per coefficient, and $O\left(N^{2}\right)$ for the complete product.

Alternatively, by using $A_{i j}=\left|\sum_{k=1}^{M} \overline{d_{k i}} d_{k j}\right|^{2}$ from (10) and manipulating the sums, one shows that the values of $y_{i}$ can be computed with the two steps

$$
c_{k l}=\sum_{j=1}^{N} d_{k j} \overline{d_{l j}} x_{j}
$$

and

$$
y_{i}=\sum_{k=0}^{M} \sum_{l=0}^{M} \overline{d_{k i}} d_{l i} c_{k l}
$$


To compute $\mathbf{A x}, c_{k l}$ is computed for each pair $(k, l)$, for a cost of $O(N)$ each. Then, each $y_{i}$ obtained by a double sum with a cost of $O\left(M^{2}\right)$ each. The total cost is thus $O\left(N M^{2}\right)$. Moreover, computations involve coefficients of the $M \times N$ matrix $\mathbf{D}$ only, making the storage of the $N \times N$ matrix A unnecessary.

The product by $\mathbf{A}_{\mathrm{dr}}$ is obtained by setting $c_{k k}=0$.

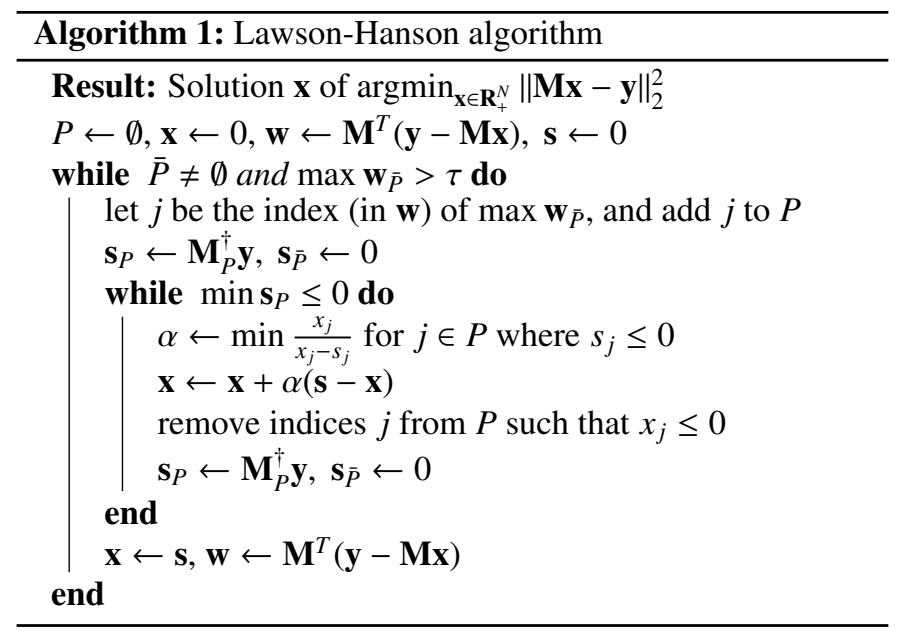

\section{References}

[1] T. F. Brooks, W. M. Humphreys, A deconvolution approach for the mapping of acoustic sources (DAMAS) determined from phased microphone arrays, J. Sound Vib. 294 (4) (2006) 856-879. doi:10.1016/j.jsv.2005.12.046.

[2] Q. Leclère, A. Pereira, C. Bailly, J. Antoni, C. Picard, A unified formalism for acoustic imaging based on microphone array measurements, Int. J. Aeroacoustics 16 (4-5) (2017) 431-456. doi:10.1177/1475472X17718883.

[3] T. Padois, A. Berry, Two and Three-Dimensional Sound Source Localization with Beamforming and Several Deconvolution Techniques, Acta Acustica united with Acustica 103 (2017) 10.

[4] F. R. d. Amaral, C. do Carmo Pagani Junior, M. A. F. d. Medeiros, Improvements in closed-section wind-tunnel beamforming experiments of acoustic sources distributed along a line, Appl. Acoust. 156 (2019) 336350. doi:10.1016/j.apacoust.2019.07.022.

[5] G. Herold, E. Sarradj, Performance analysis of microphone array methods, J. Sound Vib. 401 (2017) 152-168. doi:10.1016/j.jsv.2017.04.030.

[6] T. F. Bergh, Deconvolution approach to the mapping of acoustic sources with matching pursuit and matrix factorization, J. Sound Vib. 459 (2019) 114842. doi:10.1016/j.jsv.2019.07.008.

[7] X. Zhang, Z. Chu, Y. Yang, S. Zhao, Y. Yang, An Alternative Hybrid Time-Frequency Domain Approach Based on Fast Iterative Shrinkage-Thresholding Algorithm for Rotating Acoustic Source Identification, IEEE Access 7 (2019) 59797-59805. doi:10.1109/ACCESS.2019.2915001.

[8] W. Ma, X. Liu, Improving the efficiency of DAMAS for sound source localization via wavelet compression computational grid, J. Sound and Vibration 395 (2017) 341-353. doi:10.1016/j.jsv.2017.02.005.

[9] T. Yardibi, J. Li, P. Stoica, L. N. Cattafesta, Sparsity constrained deconvolution approaches for acoustic source mapping, The J. Acoust. Soc. of Am. 123 (5) (2008) 2631-2642. doi:10.1121/1.2896754.

[10] W. Ma, X. Liu, Compression computational grid based on functional beamforming for acoustic source localization, Appl. Acoust. 134 (2018) 75-87. doi:10.1016/j.apacoust.2018.01.006.

[11] W. Ma, X. Liu, DAMAS with compression computational grid for acoustic source mapping, J. Sound Vib. 410 (2017) 473-484. doi:10.1016/j.jsv.2017.03.027.
[12] C. J. Bahr, W. M. Humphreys, D. Ernst, T. Ahlefeldt, C. Spehr, A. Pereira, Q. Leclère, C. Picard, R. Porteous, D. Moreau, J. R. Fischer, C. J. Doolan, A Comparison of Microphone Phased Array Methods Applied to the Study of Airframe Noise in Wind Tunnel Testing, in: 23rd AIAA/CEAS Aeroacoustics Conference, American Institute of Aeronautics and Astronautics. doi: 10.2514/6.2017-3718.

[13] R. Dougherty, Extensions of DAMAS and Benefits and Limitations of Deconvolution in Beamforming, in: 11th AIAA/CEAS Aeroacoustics Conference, Aeroacoustics Conferences, American Institute of Aeronautics and Astronautics, 2005. doi:10.2514/6.2005-2961.

[14] S. Boyd, L. Vandenberghe, Convex Optimization, Cambridge University Press, USA, 2004.

[15] C. L. Lawson, R. J. Hanson, Solving Least Squares Problems, Society for Industrial and Applied Mathematics, 1995. doi:10.1137/1.9781611971217.

[16] P. Richtárik, M. Takáč, Iteration complexity of randomized blockcoordinate descent methods for minimizing a composite function, Math. Program 144 (1) (2014) 1-38. doi:10.1007/s10107-012-0614-z. URL https : //doi.org/10.1007/s10107-012-0614-z

[17] G. Chardon, gilleschardon/DAMAS, doi:10.5281/zenodo.4609267 https://zenodo.org/record/4609267 last accessed 16/03/2021.

[18] I. Schur, Bemerkungen zur Theorie der beschränkten Bilinearformen mit unendlich vielen Veränderlichen, J. Reine Angew. Math. (140) (1911) 128.

[19] C. S. Ballantine, On the Hadamard product, Math. Zeitschrift 105 (5) (1968) 365-366. doi:10.1007/BF01110298.

[20] G. H. Golub, C. F. Van Loan, Matrix Computation, 4th Edition, The Johns Hopkins University Press, Baltimore, 2013.

[21] T. Hohage, H.-G. Raumer, C. Spehr, Uniqueness of an inverse source problem in experimental aeroacoustics, Inverse Probl. 36 (7) (2020) 075012, publisher: IOP Publishing. doi:10.1088/1361-6420/ab8484.

[22] A. M. Bruckstein, M. Elad, M. Zibulevsky, On the Uniqueness of Nonnegative Sparse Solutions to Underdetermined Systems of Equations, IEEE Trans. on Inf. Theory 54 (11) (2008) 4813-4820. doi:10.1109/TIT.2008.929920

[23] M. Slawski, M. Hein, Non-negative least squares for high-dimensional linear models: Consistency and sparse recovery without regularization, Electron. J. Stat. 7 (2013) 3004-3056. doi:10.1214/13-EJS868.

[24] K. Ehrenfried, L. Koop, Comparison of Iterative Deconvolution Algorithms for the Mapping of Acoustic Sources, AIAA J. 45 (7) (2007) 1584-1595, publisher: American Institute of Aeronautics and Astronautics. doi: $10.2514 / 1.26320$.

[25] G. Chardon, F. Ollivier, J. Picheral, Localization of sparse and coherent sources by orthogonal least squares, The J. Acoust. Soc. Am. 146 (6) (2019) 4873-4882. 